\title{
OPEN Pleiotropic genetic influence on birth weight and childhood obesity
}

\author{
Suvo Chatterjee, Marion Ouidir \& Fasil Tekola-Ayele
}

Childhood obesity is a global public health problem. Understanding the molecular mechanisms that underlie early origins of childhood obesity can facilitate interventions. Consistent phenotypic and genetic correlations have been found between childhood obesity traits and birth weight (a proxy for in-utero growth), suggesting shared genetic influences (pleiotropy). We aimed to (1) investigate whether there is significant shared genetic influence between birth weight and childhood obesity traits, and (2) to identify genetic loci with shared effects. Using a statistical approach that integrates summary statistics and functional annotations for paired traits, we found strong evidence of pleiotropy $\left(P<3.53 \times 10^{-127}\right)$ and enrichment of functional annotations $\left(P<1.62 \times 10^{-39}\right)$ between birth weight and childhood body mass index (BMI)/obesity. The pleiotropic loci were enriched for regulatory features in skeletal muscle, adipose and brain tissues and in cell lines derived from blood lymphocytes. At 5\% false discovery rate, 6 loci were associated with birth weight and childhood BMI and 13 loci were associated with birth weight and childhood obesity. Out of these 19 loci, one locus (EBF1) was novel to childhood obesity and one locus (LMBR1L) was novel to both birth weight and childhood BMI/obesity. These findings give evidence of substantial shared genetic effects in the regulation of both fetal growth and childhood obesity.

Childhood overweight or obesity is a global public health problem ${ }^{1-3}$. Complex interactions of genetic susceptibility $^{4-6}$, environmental exposures ${ }^{7,8}$ and in-utero programming ${ }^{9-12}$ contribute to childhood obesity. Fetal growth and development during the intrauterine period shape physiological and structural processes that impact risk for childhood obesity $(\mathrm{COB})^{9}$. Birth weight, a marker of fetal growth and prenatal environment, has been consistently associated with $\mathrm{COB}^{13-20}$. Notably, a significant positive genetic correlation has been found between birth weight and $\mathrm{COB} /$ childhood body mass index $(\mathrm{CBMI})^{21}$, suggesting the possibility for genetic links these phenotypes may share. Insight into the shared genetic underpinnings of these early life phenotypes facilitates knowledge on the potential common molecular pathways and intervention targets of abnormal birth weight and COB/CBMI. However, pleiotropic evidence on these traits remains scarce.

Recent genome wide association studies (GWAS) have reported that shared genetic effects (pleiotropy) might explain a substantial proportion of correlations between complex human traits ${ }^{22-26}$. In an analysis of the GWAS catalog $^{27}$ it was found that $16.9 \%$ of the reported genes were associated with multiple traits ${ }^{28}$. With the advent of large scale independent GWASs on multiple early life traits and functional annotation database ${ }^{29-31}$, innovative statistical approaches can be applied to identify and test for novel pleiotropic loci.

In addition, accounting for shared genetic effects improves statistical power for detecting genetic variants that are associated with complex traits ${ }^{32}$. Both birth weight and COB/CBMI are under strong genetic control ${ }^{10,11,33-35}$, with relatively high heritability estimates of $25-40 \%$ for birth weight ${ }^{35}$ and $30-70 \%$ for COB $/ \mathrm{CBMI}^{34}$. However, the heritability of the traits explained by genetic variants identified using published GWAS for birth weight ${ }^{36}$, $\mathrm{COB}^{37}$ and $\mathrm{CBMI}^{38-40}$ was less than $10 \%$. Therefore, detecting novel genetic loci via pleiotropic analysis can help close the gap in missing heritability.

To date, pleiotropic associations of birth weight and COB/CBMI have not been exclusively investigated. In the current study, we used an innovative statistical approach that integrates pleiotropy and functional annotation data with the following aims: (1) to investigate whether there is significant shared genetic influence between birth weight and $\mathrm{COB} / \mathrm{CBMI}$ (pleiotropy enrichment test), (2) to examine whether the shared risk variants are functionally more enriched in birth weight and COB/CBMI as compared to only considering single traits in models that incorporate a variety of genetic annotations in different tissues and cell types (annotation enrichment test),

Epidemiology Branch, Division of Intramural Population Health Research, Eunice Kennedy Shriver National Institute of Child Health and Human Development, National Institutes of Health, 6710B Rockledge Drive, Room 3204, Bethesda 20892-7004, USA. ${ }^{\circledR}$ email: ayeleft@mail.nih.gov 


\begin{tabular}{|c|c|c|c|c|c|}
\hline \multirow[b]{2}{*}{ Trait pair } & \multirow[b]{2}{*}{ Annotation } & \multicolumn{2}{|l|}{ Genetic pleiotropy } & \multicolumn{2}{|c|}{$\begin{array}{l}\text { Functional annotation } \\
\text { enrichment }\end{array}$} \\
\hline & & Enrichment fold (s.e) & $P$ value & q11/q00 (s.e) & $P$ value \\
\hline \multirow{4}{*}{$\mathrm{BW}_{\mathrm{EU}}$ and CBMI } & CADD & $2.683(0.05)$ & $1.17 \times 10^{-277}$ & $1.69(0.08)$ & $2.69 \times 10^{-38}$ \\
\hline & eQTLs & $2.679(0.05)$ & $1.18 \times 10^{-277}$ & $2.10(0.04)$ & $\leq 1 \times 10^{-300}$ \\
\hline & DHSs & $2.67(0.05)$ & $2.52 \times 10^{-276}$ & $1.17(0.01)$ & $\leq 1 \times 10^{-300}$ \\
\hline & TFBS & $2.685(0.05)$ & $1.18 \times 10^{-277}$ & $1.09(0.03)$ & 0.03 \\
\hline \multirow{4}{*}{$\mathrm{BW}_{\mathrm{EU}}$ and $\mathrm{COB}$} & CADD & $5.413(0.19)$ & $9.79 \times 10^{-144}$ & $1.54(0.18)$ & $1.42 \times 10^{-27}$ \\
\hline & eQTLs & $5.396(0.29)$ & $9.79 \times 10^{-144}$ & $1.73(0.09)$ & $\leq 1 \times 10^{-300}$ \\
\hline & DHSs & $5.379(0.24)$ & $1.45 \times 10^{-143}$ & $1.15(0.03)$ & $\leq 1 \times 10^{-300}$ \\
\hline & TFBS & $5.383(0.24)$ & $9.79 \times 10^{-144}$ & $1.01(0.06)$ & 0.21 \\
\hline \multirow{4}{*}{$\mathrm{BW}_{\mathrm{TR}}$ and $\mathrm{CBMI}$} & CADD & $2.653(0.05)$ & $9.97 \times 10^{-274}$ & $1.79(0.08)$ & $1.62 \times 10^{-39}$ \\
\hline & eQTLs & $2.648(0.05)$ & $9.98 \times 10^{-271}$ & $2.15(0.04)$ & $\leq 1 \times 10^{-300}$ \\
\hline & DHSs & $2.641(0.05)$ & $2.18 \times 10^{-269}$ & $1.17(0.02)$ & $\leq 1 \times 10^{-300}$ \\
\hline & TFBS & $2.655(0.05)$ & $9.98 \times 10^{-271}$ & $1.10(0.03)$ & 0.01 \\
\hline \multirow{4}{*}{$\mathrm{BW}_{\mathrm{TR}}$ and $\mathrm{COB}$} & CADD & $5.209(0.17)$ & $3.51 \times 10^{-127}$ & $1.68(0.18)$ & $1.94 \times 10^{-29}$ \\
\hline & eQTLs & $5.204(0.18)$ & $3.51 \times 10^{-127}$ & $1.80(0.10)$ & $\leq 1 \times 10^{-300}$ \\
\hline & DHSs & $5.186(0.20)$ & $6.52 \times 10^{-127}$ & $1.12(0.03)$ & $\leq 1 \times 10^{-300}$ \\
\hline & TFBS & $5.197(0.18)$ & $3.51 \times 10^{-127}$ & $0.99(0.07)$ & 0.04 \\
\hline
\end{tabular}

Table 1. Genetic pleiotropy and enrichment of functional deleteriousness among genetic loci associated with birth weight and childhood obesity traits. $q 11 / q 00$ is the ratio of the probability of jointly associated SNPs being functionally annotated to the probability of a null SNP (not associated with either trait) being functionally annotated. $B W_{E U}$ and $C B M I$ European birth weight and childhood body mass index, $B W_{E U}$ and $C O B$ European birth weight and childhood obesity, $B W_{T R}$ and $C B M I$ trans-ethnic birth weight and childhood body mass index, $B W_{T R}$ and $C O B$ trans-ethnic birth weight and childhood obesity, $C A D D$ combined annotation dependent depletion, eQTLs expression quantitative loci, DHSs DNase I hypersensitivity sites, TFBS transcription factor binding sites.

and (3) to identify genetic loci with shared genetic effects on birth weight and COB/CBMI. Our analysis found abundant evidence of pleiotropy and significant enrichment of functional annotations for shared risk variants associated with birth weight and COB/CBMI. We identified genetic loci with overlapping influence on both birth weight and COB/CBMI. Most of those loci have been associated with childhood BMI/obesity in previous GWAS but not with birth weight, whereas one locus $(E B F 1)$ was novel to childhood obesity and one locus $(L M B R 1 L)$ was novel to both birth weight and childhood BMI/obesity.

\section{Results}

Data on summary statistics including $P$ values and direction of effects in GWAS meta-analysis of birth weight, CBMI and COB was obtained from publicly available data of the Early Growth Genetics (EGG) consortium. Two sets of GWAS summary statistics were available for birth weight, one involving European ancestry individuals $\left(\mathrm{BW}_{\mathrm{EU}}\right)$ and a second from trans-ancestry meta-analysis $\left(\mathrm{BW}_{\mathrm{TR}}\right)$. The GWAS summary statistics for CBMI and $\mathrm{COB}$ were from European ancestry individuals. Therefore, tests of pleiotropy were conducted on four trait pairs: $\mathrm{BW}_{\mathrm{EU}}-\mathrm{CBMI}, \mathrm{BW}_{\mathrm{EU}}-\mathrm{COB}, \mathrm{BW}_{\mathrm{TR}^{-}}-\mathrm{CBMI}$, and $\mathrm{BW}_{\mathrm{TR}}-\mathrm{COB}$.

Pleiotropy analysis was performed via the $\mathrm{R}$ package $\mathrm{GPA}^{41}$ which implements a statistical approach to explore the genetic architecture of complex traits by integrating pleiotropy and functional annotation information, including prioritizing risk genetic variants. GPA performs hypothesis test for evaluating enrichment of pleiotropy and functional annotations using a likelihood ratio test approach. For each trait pair, we performed tests of pleiotropy and enrichment of functional annotations based on 4 functional categories: combined annotation dependent depletion (CADD) (http://www.cadd.gs.washington.edu) ${ }^{42}$, expression quantitative trait loci (eQTL) (https:// gtexportal.org/home/datasets) ${ }^{43}$, transcription factor binding sites (TFBS) (ftp://ccg.epfl.ch/snp2tfbs/mapped_ files/annotated) ${ }^{44}$, and DNase I hypersensitivity sites (DHS) (https://github.com/joepickrell/1000-genomes) ${ }^{45}$.

Evidence of pleiotropy between birth weight and childhood obesity traits. Under each functional category, we found evidence of significant pleiotropic genetic effects for all tested trait pairs. Pleiotropic genetic effects had enrichment fold ranging from 2.6 to $5.4\left(1.17 \times 10^{-277}<P<3.51 \times 10^{-127}\right)$ under CADD annotation, 2.6 to $5.2\left(1.18 \times 10^{-277}<P<3.51 \times 10^{-127}\right)$ under eQTL annotation, 2.6 to $5.1\left(2.52 \times 10^{-276}<P<6.52 \times 1\right.$ $\left.0^{-127}\right)$ under DHS annotation, and 2.6 to $5.1\left(1.18 \times 10^{-277}<P<6.52 \times 10^{-127}\right)$ under TFBS annotation (Table 1).

Enrichment of functional annotations. Next, we assessed whether genetic loci with regulatory functional annotations had higher likelihood of being associated with both birth weight and CBMI/COB as compared to genetic loci without functional annotations. Functional annotation of the 2.4 million single-nucleotide polymorphisms (SNPs) tested was done using CADD ${ }^{42}$, eQTL ${ }^{43}$, TFBS $^{44}$, and DHS ${ }^{45}$. Out of the $\sim 2.4$ million SNPs tested, approximately $2 \%$ of the SNPs were annotated as CADD related, $7 \%$ as eQTL related, $20 \%$ as TFBS 
A.BW $\mathrm{EU}_{\mathrm{U}}$ and $\mathrm{CBMI}$

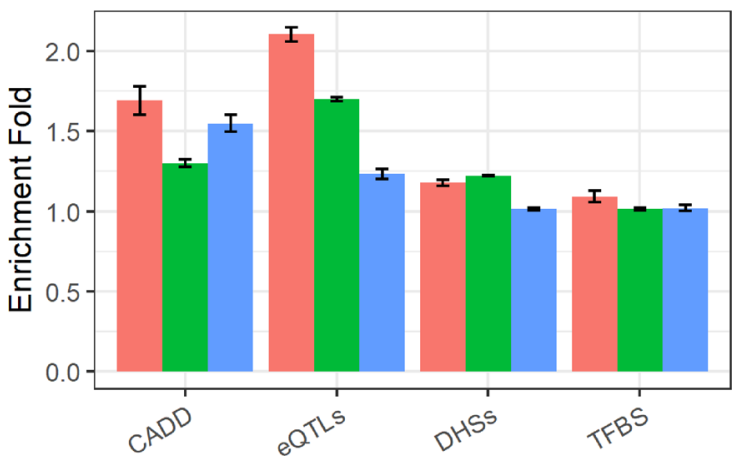

Annotations

\section{C.BW $\mathrm{TR}_{\mathrm{R}}$ and $\mathrm{CBMI}$}

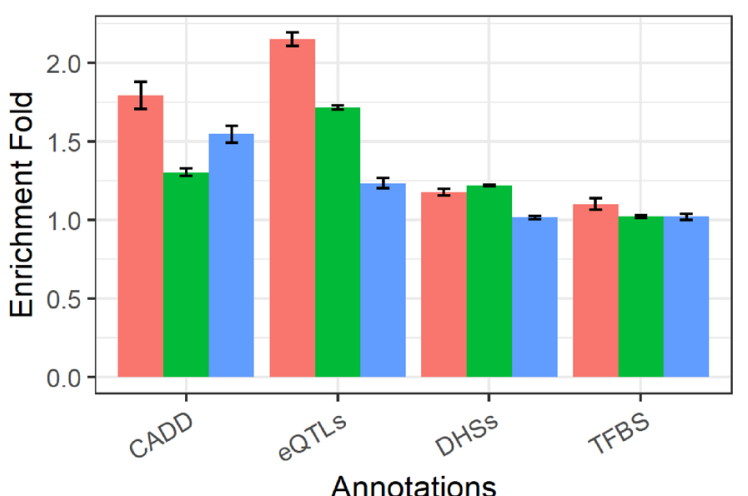

Traits
Annotations
B.BW $\mathrm{EU}_{\mathrm{U}}$ and $\mathrm{COB}$

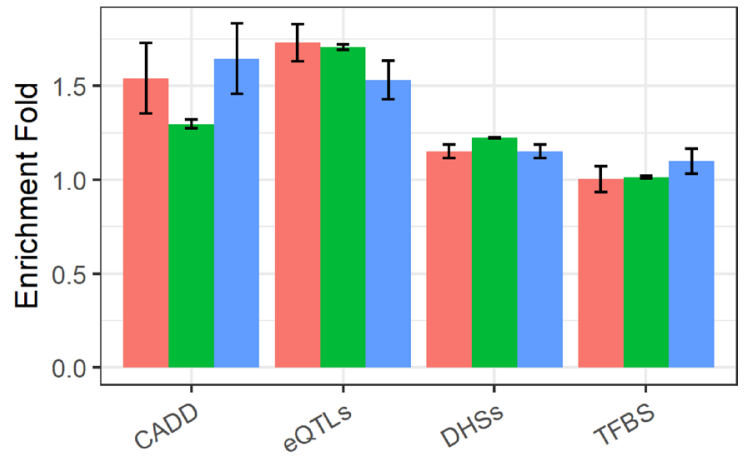

Annotations

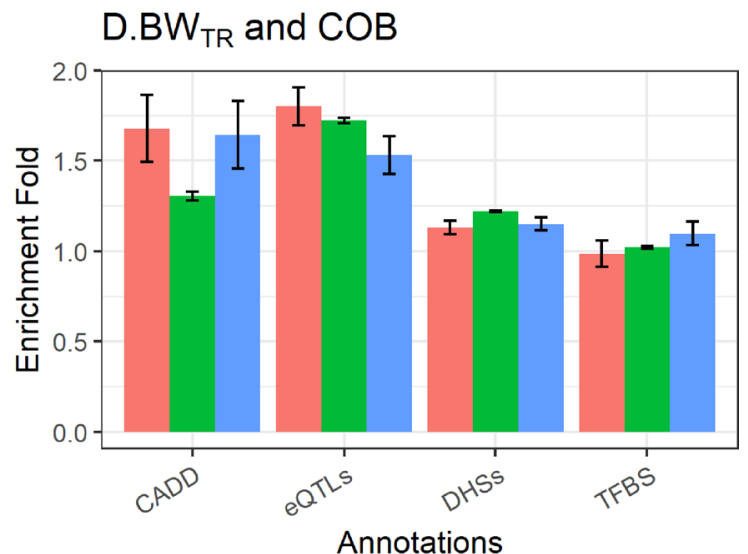

Childhood obesity traits only (q10/q00)

Figure 1. Enrichment of four functional annotations among loci jointly associated with birth weight and childhood obesity traits. (A) European birth weight and childhood body mass index (BW $\mathrm{EU}^{-C B M I),(B)}$ European birth weight and childhood obesity (BW $\left.\mathrm{EU}^{-} \mathrm{COB}\right),(\mathrm{C})$ Trans-ethnic birth weight and childhood body mass index (BW $\left.\mathrm{TR}_{\mathrm{TR}}-\mathrm{CBMI}\right)$, (D) Trans-ethnic birth weight and childhood obesity $\left(\mathrm{BW}_{\mathrm{TR}^{-}} \mathrm{COB}\right)$. The bars denote the enrichment fold for variants that are associated jointly with BW and childhood obesity traits (red), only with birth weight (green), and only with childhood obesity traits (blue), respectively.

related and $51 \%$ as DHS related (Table S1). Enrichment of each of the four functional categories was tested for four trait pairs using the GPA model ${ }^{41}$, resulting in 16 tests of functional enrichment.

To compare the functional deleteriousness of the pleiotropic SNPs with non-pleiotropic SNPs, each variant in our analysis was annotated to be deleterious or non-deleterious based on CADD score $\geq 15$ or CADD score $<15$, respectively ${ }^{42}$. Out of the 16 tests, SNPs associated with pairs of birth weight and childhood obesity traits were more likely to be functionally deleterious than SNPs associated with neither trait in 15 tests (enrichment fold ranging from 0.99 to 2.15 and Bonferroni corrected $P$ values ranging from $\left.1 \times 10^{-300} \leq P<1.62 \times 10^{-39}\right)$; however, 1 test under TFBS category for $\mathrm{BW}_{\mathrm{EU}}$-COB pair was not significant $(P=0.21)$ (Table 1$)$. Enrichment of eQTL annotation was consistently higher for SNPs associated with both birth weight and COB traits compared to SNPs associated with only birth weight or CBMI/COB (Fig. 1).

We further examined the specific tissues, cell types and transcription factors that had relatively higher functional enrichment among the common variants between birth weight and childhood obesity traits. For each trait pair, we performed annotation enrichment tests of 49 tissues with eQTL annotations ${ }^{43}, 402$ tissues/cell lines/cell types with DHSs annotations ${ }^{45}$, and 195 transcription factors in the TFBS database ${ }^{44}$, one at a time. The most significant eQTL enrichments $(P<0.05$ and lowest in the joint trait association) were observed in tissues from skeletal muscle, adipose, brain, heart, esophagus, thyroid, adrenal, colon, small intestine and whole blood. The most significant DHS enrichments were observed in cell lines derived from blood lymphocytes (e.g. CD4+), skin (e.g. iPS), cancer (e.g. HeLa), embryo (e.g. embryonic stem cells) and in tissues from fetal brain. The most significant transcription factor enrichments were Interferon Regulatory Factor 1, AT-Rich Interaction Domain $3 \mathrm{~A}$ and Testicular receptor 4 (Table S2-S3).

At 5\% false discovery rate, 19 loci (consisting 509 SNPs, with each locus having correlated SNPs with linkage disequilibrium $\mathrm{r}^{2}>0.5$ ) were jointly associated with birth weight and childhood obesity traits (Figs. S1-S4). Among these 19 loci, 5 loci were jointly associated with European BW and COB traits, 1 locus was jointly associated with transethnic BW and $\mathrm{COB}$ traits and 13 loci were jointly associated with both European and transethnic BW and COB traits (Table S4-S5). Additionally, out of these 19 loci, 17 loci were novel to birth weight but not 

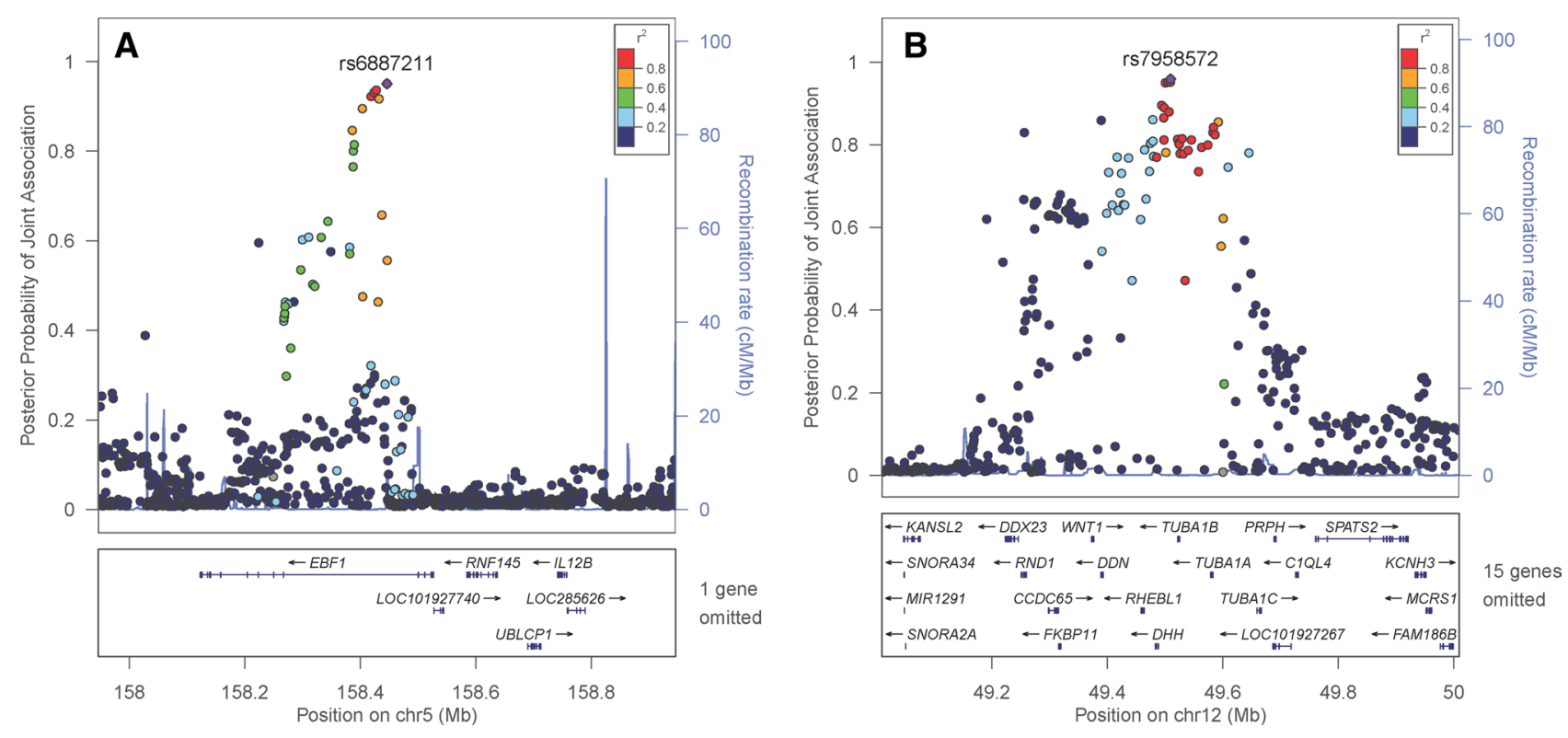

Figure 2. Regional plots of novel pleiotropic loci associated with birthweight and childhood obesity traits. (A) rs6887211 (EBF1) locus association with both birth weight and childhood body mass index (B) rs7958572 (LMRB1L) locus associated with both birthweight and childhood obesity or childhood body mass index. The horizontal axes cover a region $500 \mathrm{~kb}$ upstream and downstream from the reference SNP. The vertical axes denote the joint association probabilities of the SNPs with both birthweight and childhood obesity traits. The purple triangles denote the index SNPs (rs6887211 in EBF1 and rs7958572 in LMRB1L). All other colored points denote the surrounding SNPs in that region, and they are colored based on their linkage disequilibrium $\left(\mathrm{r}^{2}\right)$ with the reference SNP. The box at the bottom shows genes that fall in the region.

childhood obesity traits at the genome-wide significance level $\left(P<5 \times 10^{-8}\right.$, in the NHGRI-EBI GWAS catalogue: www.ebi.ac.uk/gwas/), one locus (rs6887211 in EBF1) was novel to childhood obesity traits but not birth weight, and one locus (rs7958572 $4 \mathrm{~Kb}$ upstream to $L M B R 1 L$ ) was novel to both birth weight and childhood obesity traits (Fig. 2). The EBF1 locus was only suggestively associated with childhood obesity traits $\left(P=8.44 \times 10^{-5}\right)$ and the $L M B R 1 L$ locus was only suggestively associated with birth weight $\left(P=2.4 \times 10^{-3}\right)$ and childhood obesity traits $\left(P=7.3 \times 10^{-6}\right)$ in previous $\mathrm{GWAS}^{36,39}$.

For most of the jointly associated loci, the alleles associated with decreased birth weight were associated with lower CBMI/COB risk. In contrast, for three loci (EBF1, NCOA1 and SEC16B), nearly all alleles associated with decreased birth weight were associated with higher CBMI/COB risk (Table S4-S5). Furthermore, we evaluated our findings using the European-ancestry summary statistics in a recent childhood obesity GWAS ${ }^{46}$. Out of the 342 SNPs in our study found to be associated with both birthweight and childhood obesity, $98.5 \%$ reached $>95 \%$ posterior probability when we used this validation childhood obesity GWAS (Table S6).

Pathway analysis. To further understand the functional relevance of the genetic loci jointly associated with birth weight and childhood obesity traits, we performed pathway enrichment analysis of the 25 genes that are near the pleiotropic SNPs using the Ingenuity Pathway Analysis (IPA) tool (QIAGEN Inc, https://digitalins ights.qiagen.com/). The top enriched IPA Canonical Pathways included RAR Activation and Estrogen Receptor Signaling (Table S7) and the top enriched IPA Disease or Function annotations included brain size and morphology (Table S8).

Colocalization analysis. To determine whether the signals identified represent horizontal pleiotropy (influence birthweight and childhood obesity via independent biological pathways) or vertical pleiotropy (influence via shared mechanism in a causal pathway) we performed colocalization analysis using the coloc $\mathrm{R}$ package $^{47}$. Only the EBF1 locus showed evidence of colocalization with a posterior probability of 0.87 for being associated with both traits and share a single causal variant (Tables S9 and S10).

\section{Discussion}

There is growing evidence that several genetic variants can influence two or more traits ${ }^{28,48,49}$. Investigating such effects in early life traits can facilitate our understanding of their complex genetic architecture and in developing early life interventions to promote long-term health ${ }^{50,51}$. The present study revealed substantial genetic pleiotropic effects between birth weight and childhood obesity traits. We also found that biologically functional SNPs are more likely to be associated with both birth weight and COB traits compared to SNPs that are not functional, consistent with evidence of high evolutionary conservation of pleiotropic genes and their consequences ${ }^{52}$. Lastly, we identified 19 genetic loci with pleiotropic effects, including loci in EBF1 and LMRBL1 that have not been 
associated with birth weight and/or COB phenotypes in previous GWAS. With the majority (68.4\%) of the 19 loci reaching the significance threshold in analyses involving European-only or trans-ethnic birthweight GWAS and the remaining loci exhibiting high posterior probability of association (0.85-0.93), the results suggest that the effects of many pleiotropic loci are likely shared across ancestries. In all, these findings facilitate our understanding of the genetic mechanism that may underlie associations of early life growth with COB traits.

The birth weight-decreasing allele of the novel pleiotropic SNP rs6887211 (EBF1) was found to be associated with increased risk of $C O B$ traits. The association of $E B F 1$ with birth weight and gestational duration is well-recognized ${ }^{36,53-55}$. EBF1 is highly expressed in adipocytes (Fig. S5) and plays a crucial role in adipogenesis and development of B lymphocytes. Dysregulated expression of $E B F 1$ is associated with adipose hypertrophy, adipose inflammation, variation in body fat distribution and altered adipose morphology through impaired adipogenesis, which in turn has been implicated as a key factor in the development of obesity related traits ${ }^{56-59}$. $E B F 1$ has a well-known association with cardiometabolic traits in adults. SNPs near EBF1 have been associated with blood pressure ${ }^{60}$ and hip circumference ${ }^{61}$. Notably, a multi-ethnic GWAS in adults has found an association between rs1650505 (near EBF1) and pericardial adipose tissue volume ${ }^{56}$. Previous studies found substantial overlaps between childhood and adult obesity GWAS loci62. While there is relatively weak LD between the lead SNP showing pleiotropic effect in our study (rs6887211) and $r s 1650505\left(\mathrm{r}^{2}=0.06\right.$ in CEU), we acknowledge that the novelty of the locus in childhood BMI may be in part due to the small study power of the source GWAS used for childhood BMI. Furthermore, our novel SNP and its LD proxies $\left(\mathrm{r}^{2} \geq 0.8\right)$ overlapped with DNase I hypersensitivity sites of blood lymphocytes and fetal muscle further indicating that many components of the metabolic and inflammatory pathways are positively and directly regulated by EBF1 as emphasized in a prior study ${ }^{63}$. These findings collectively indicate EBF1's role in fetal growth and general adiposity which highlights that it should be considered as a key player in understanding the association of early life growth with the development of COB.

The birth weight-decreasing allele of the novel pleiotropic SNP in LMBR1L (rs7958572) was found to be associated with decreased risk of COB. $L M B R 1 L$ has regulatory effects on the canonical Wnt signaling pathway. It stabilizes the beta-catenin destruction complex ${ }^{64}$ that is required for regulating CTNNB1 expression, and is responsible in making the beta-catenin protein which plays a key role in the canonical Wnt signaling pathway ${ }^{65-68}$. The Wnt signaling pathway is conserved in various organisms and plays important roles in development, cellular proliferation, and differentiation ${ }^{69}$. Previous studies have demonstrated that the Wnt signaling pathway regulates adipogenesis and maintains the undifferentiated state of pre-adipocytes by inhibiting adipogenic gene expression ${ }^{70,71}$. In addition, the Wnt signaling cascade plays fundamental roles in placental development by regulating trophoblast differentiation and invasion, and aberrant Wnt signaling activation can have downstream consequences on fetal growth ${ }^{72-74}$. Our finding here for $L M B R 1 L$, together with the regulatory impact of $L M B R 1 L$ on Wnt signaling and the functional relevance of the Wnt pathway in placental function, suggests a novel hypothetical mechanism by which $L M B R 1 L$ contributes to the link between fetal growth and development of COB. Future studies such as eQTLs in tissues relevant to early life traits can facilitate better inference from the pleiotropic SNP to a potential functional gene.

In agreement with previous observational studies which reported that small for gestational age (SGA) or low birth weight is associated with both lower and higher risk of $\mathrm{COB}^{13-20}$, we observed that birth weight-decreasing alleles of pleiotropic SNPs may be associated with either increased or decreased risk of COB. We found that the birth weight-reducing alleles of most of the pleiotropic loci we found (e.g. FTO, FAIM2, TMEM18) have previously been associated with decreased risk of COB, suggesting that concurrent genetic mechanisms may play an important role in the well-recognized positive correlation between low birth weight and low risk of COB. We also found that in 3 out of our 19 pleiotropic loci (i.e., EBF1, SEC16B, NCOA1) the birth weight-decreasing alleles were associated with increased risk of COB. Genetic loci near EBF1and SEC16B genes have been associated with birth weight, SGA and adiposity traits ${ }^{53-56,75,76}$; however, the association of NCOA1 with birth weight or COB traits remains elusive. Interestingly, co-activators like NCOA1 are fundamental in uterine growth by regulating placental morphogenesis, embryo survival and interacting with estrogen receptors in the human placenta to enhance estrogen signaling which has downstream consequences on birth weight ${ }^{77,78}$. NCOA1 is also highly expressed in the hypothalamus region of the brain (Fig. S6) which is implicated in appetite control, weight loss and shaping the metabolic landscape of an individual ${ }^{79}$ and any disruption of NCOA1's function can consequently lead to several metabolic disorders which may explain its association with $\mathrm{COB}^{79,80}$. Therefore, the mixed direction of effects found in our study highlights the complex relationship of in-utero growth and COB traits and may provide new scopes to understand the mechanisms of development of COB.

Functional enrichment tests and pathway analysis revealed three key biological processes that may jointly contribute to the association of early life growth and development of COB. Our study found that SNPs associated with both birth weight and $\mathrm{COB}$ traits mapped to genes that were significantly enriched in immune system pathways and regulated the signaling of immune cells and estrogen. Estrogen plays an essential role in regulating immune response, through its interactions with the receptors on the immune cells and their functioning ${ }^{81}$. Previous studies ${ }^{82,83}$ have demonstrated that the cell-mediated immune response is impaired in obese children along with an over-representation of the immune and inflammatory response in adipose tissues of children. We also found the pleiotropic SNPs to be significantly enriched in Retinoic Acid (RA) signaling pathway and have cis-regulatory effects on gene expression in adipose tissue and muscle skeletal. Multiple studies have documented that RA regulates adipogenesis ${ }^{84-86}$. RA acts as a high affinity ligand for the nuclear receptor peroxisome proliferation-activated receptor $\beta / \delta$ which is a master regulator of lipid metabolism and glucose homeostasis. Deactivation of these receptors decreases lipid catabolism in adipose tissue and skeletal muscle, increasing the risk of obesity. Lastly, we found the pleiotropic SNPs to be significantly enriched in intracerebral signaling pathway by having regulatory effects on several cerebral tissues. Brain-derived neurotrophic factor (BDNF), a key neurotrophin with multipotent impact on brain signaling ${ }^{87}$ plays an important role in regulating energy homeostasis and metabolism ${ }^{88-90}$. BDNF acts on hypothalamic PVN and VMH neurons to suppress appetite 
and mediate the anorexigenic effects of $\mathrm{MSH}$ acting on the MCH-4 receptor ${ }^{89}$. Deficit in BDNF levels or signaling is attributed to the development of obesity ${ }^{91,92}$, indicating that pathways of intracerebral signaling may be related to $\mathrm{COB}$. Our results suggest that these same causal biologic pathways likely influence early life growth, but further research is needed to understand how these pathways that influence COB also influence birth weight.

We recognize that our study has limitations. First, despite the large sample sizes of the consortia-based metaanalysis GWAS studies included in our study, there were differences in sample sizes and number of SNPs among the various GWAS studies. These contrasts may contribute to power differences in identifying pleiotropic loci. Additionally, some of the observed associations might be due to independent associations of the locus on birth weight and $\mathrm{COB}$ traits, due to the correlation of the traits in a causal pathway or some unmeasured characteristics. Second, the inference on joint associations of the variants in our study is based on estimates from the GPA model which performs optimally under low to moderate genetic correlation between the traits; however, under complex and weaker correlations the estimates of the GPA model can be biased. Third, some cohorts contributed to both childhood BMI and birthweight GWAS. Since GPA does not account for sample overlaps, we do not know whether the estimates are biased. Fourth, our analysis did not find functional enrichment of the pleiotropic loci between birth weight and COB under the TFBS annotation set, which requires further investigation in larger samples. An important strength of our study is the integrated modelling of functional annotations and GWAS summary statistics data from pairs of traits. This multi-trait approach has been most conducive in testing for functional enrichment and identifying novel genetic loci with their shared impacts on multiple traits, expanding our understanding of the genetic links between fetal growth and COB traits.

In conclusion, this study found that pleiotropic genetic influences and enrichment of functional annotations are substantially pervasive in the genetic architectures of birth weight and COB traits. The novel loci found in the analysis and the pathways through which the associated genes act have the potential to unravel the genetic basis that underlines associations between early growth and development of COB.

\section{Materials and methods}

Birth weight, childhood BMI and childhood obesity GWAS summary statistic data. Summary statistic data including $P$ values and direction of effects in GWAS meta-analysis of birth weight ${ }^{36}, \mathrm{CBMI}^{39}$ and $\mathrm{COB}^{37}$ was obtained from the publicly available data of EGG (Table S2). The GWAS meta-analysis of CBMI was performed on 20 combined studies with a total sample size of 35,668 children. Children aged 2-10 years with European ancestry were included in the study ${ }^{39}$. The GWAS meta-analysis of COB combined 14 studies of European children aged 2-10 years with a total sample size of 5530 cases and 8318 controls. Children who had a BMI $>95$ th percentile were considered to be obese cases while children of $\mathrm{BMI}<50$ th percentile were considered to be controls ${ }^{37}$. The GWAS meta-analysis of birth weight included neonates of European ancestry $\left(\mathrm{BW}_{\mathrm{EU}}\right.$, $\mathrm{n}=298,142$ ) and transethnic (African, South Asian, European) meta-analysis $\left(\mathrm{BW}_{\mathrm{TR}} \mathrm{n}=321,223\right)$. Individuals who were part of multiple births, who reported their birth weight in multiple visits with the mean difference in their birth weight being $>1 \mathrm{~kg}$ and individuals whose birth weight was $<2.5 \mathrm{~kg}$ or $>4.5 \mathrm{~kg}$ were excluded ${ }^{36}$. The results from the GWAS of own birth weight (as opposed to offspring birth weight), and without adjustment for maternal genotype, were used in this analysis.

Functional annotation data. We used 4 annotation databases CADD ${ }^{42}, \mathrm{eQTL}^{43}, \mathrm{TFBS}^{44}$ and $\mathrm{DHSs}^{45}$ to functionally annotate the SNPs. Under CADD framework, implemented in CADD v1.2 (http://www.cadd.gs. washington.edu $)^{42}$, a deleteriousness score (combined SVM score; c-score) is generated using the integrated functional and evolutionary importance of each variant from 63 annotation sources. Phred-like scores (ranged 1-99) are further generated based on the rank of each variant relative to 8.6 billion substitutions in human reference genome $\left(-10^{\star} \log _{10}[\mathrm{rank} /\right.$ total $\left.]\right)$. Each variant in our analysis receiving a score $\geq 15$ was assigned an annotation of 1 (deleterious) while scores $<15$ were assigned an annotation of 0 (non-deleterious) ${ }^{42}$. In addition to CADD, which comprises a composite score, we used annotations from a variety of tissues and cell lines to elucidate the regulatory mechanisms of the risk variants. The eQTL annotations was obtained from dbGaP accession number phs000424.vN.pN on 02/11/20 (https://gtexportal.org/home/datasets) ${ }^{43}$ which consisted of cis-eQTL files on 49 different tissues. We took the intersection of these eQTL with the common variants of BW and COB/ CBMI and the variants that overlapped were annotated as 1 and others as 0 . The TFBS annotation (ftp://ccg.epfl. ch/snp2tfbs/mapped_files/annotated//44 had data on 195 different transcription factors. Similar to eQTL, we took the intersection of the TFBS to annotate the common variants of birth weight and COB/CBMI. The DHSs annotation data, which has also been used in other studies ${ }^{45,93}$, was downloaded from (https://github.com/joepi ckrell/1000-genomes) ${ }^{45}$ and comprised of 402 binary annotations that included maps of DNase-I hypersensitive sites from different primary tissues, cell lines and cell types ${ }^{94,95}$.

Statistical Analysis. We used a unified statistical approach that integrates summary statistics with functional annotations for paired traits using probabilistic models implemented in genetic analysis incorporating pleiotropy and annotation $(\mathrm{GPA})^{41}$. For convenience, we briefly introduce the GPA model and its notations below.

Suppose the $P$ values from two GWAS have been collected in an $\mathrm{M} \times 2$ matrix, $\mathrm{p}=\left[\mathrm{p}_{\mathrm{jk}}\right]$, where $\mathrm{p}_{\mathrm{jk}}$ denotes the $P$ value of the jth SNP in the kth GWAS, $\mathrm{k}=1,2$ (in our case) and M is the number of SNPs. In the GPA model, these $P$ values are assumed to come from a mixture of null (un-associated) and non-null (associated), with probability $\pi_{0}$ and $\pi_{1}=1-\pi_{0}$, respectively. GPA uses the Uniform distribution on $[0,1]$ and the Beta distribution with parameters $(\alpha, 1)$ to model the $P$ values from the null and non-null groups, respectively.

Let $\mathrm{Zj} \in\{00,10,01,11\}$ indicate the association between the jth SNP and the two traits: $\mathrm{Zj}=00$ means the jth $\mathrm{SNP}$ is associated with neither of them, $\mathrm{Zj}=10$ means it is only associated with the first trait, $\mathrm{Zj}=01$ means it 
is only associated with the second trait, and $\mathrm{Zj}=11$ means it is associated with both trait. Thus, the four-group model is represented as:

$$
\begin{array}{lll}
\pi_{00}=\operatorname{Pr}(\mathrm{Zj}=00): \mathrm{p}_{\mathrm{j} 1} \sim \mathrm{U}[0,1], \mathrm{p}_{\mathrm{j} 2} \sim \mathrm{U}[0,1], & \text { if } \mathrm{Zj}=10 \\
\pi_{10}=\operatorname{Pr}(\mathrm{Zj}=10): \mathrm{p}_{\mathrm{j} 1} \sim \operatorname{Beta}(\alpha 1,1), \mathrm{p}_{\mathrm{j} 2} \sim \mathrm{U}[0,1], & \text { if } \mathrm{Zj}=00 \\
\pi_{01}=\operatorname{Pr}(\mathrm{Zj}=01): \mathrm{p}_{\mathrm{j} 1} \sim \mathrm{U}[0,1], \mathrm{p}_{\mathrm{j} 2} \sim \operatorname{Beta}(\alpha 2,1), & \text { if } \mathrm{Zj}=01 \\
\pi_{11}=\operatorname{Pr}(\mathrm{Zj}=11): \mathrm{p}_{\mathrm{j} 1} \sim \operatorname{Beta}(\alpha 1,1), \mathrm{p}_{\mathrm{j} 2} \sim \operatorname{Beta}(\alpha 2,1), & \text { if } \mathrm{Zj}=11
\end{array}
$$

where $\mathrm{p}_{\mathrm{j} 1}$ and $\mathrm{p}_{\mathrm{j} 2}$ is the $P$ value of the jth SNP in GWAS 1 and 2 . GPA further incorporates functional annotation as follows. Let an $\mathrm{M}$-dimensional vector A collect functional information from an annotation source, where $\mathrm{Aj} \in\{0,1\}$ indicates whether the $\mathrm{jth}$ SNP is a functional unit according to the annotation source. For example, given an eQTL data, if the $\mathrm{jth}$ SNP is an eQTL, then $\mathrm{Aj}=1$, otherwise $\mathrm{Aj}=0$. The relationship between $\mathrm{Zj}$ and $\mathrm{Aj}$ is described as:

$$
\begin{aligned}
& \mathrm{q}_{00}=\operatorname{Pr}(A j=1 \mid \mathrm{Zj}=00), \\
& \mathrm{q}_{10}=\operatorname{Pr}(A j=1 \mid \mathrm{Zj}=10), \\
& \mathrm{q}_{01}=\operatorname{Pr}(A j=1 \mid \mathrm{Zj}=01), \\
& \mathrm{q}_{11}=\operatorname{Pr}(A j=1 \mid \mathrm{Zj}=11),
\end{aligned}
$$

where $\mathrm{q}_{00}$ is the probability of a null SNP being annotated, $\mathrm{q}_{10}$ is the probability of the first trait-associated SNP being annotated, $\mathrm{q}_{01}$ is the probability of the second trait-associated SNP being annotated, and $\mathrm{q}_{11}$ is the probability of jointly associated SNP being annotated. GPA then implements an efficient EM-algorithm to obtain the estimates of the model parameters: $\left\{\pi_{00}, \pi_{10}, \pi_{01}, \pi_{11}, \mathrm{q}_{00}, \mathrm{q}_{10}, \mathrm{q}_{01}, \mathrm{q}_{11}, \alpha\right\}$.

To assess the significance of enrichment for pleiotropy between two traits it uses the likelihood ratio test (LRT) with $\mathrm{H}_{0}: \pi_{11}=\left(\pi_{10}+\pi_{11}\right)\left(\pi_{01}+\pi_{11}\right)$, versus $\mathrm{H}_{1}$ : not H0. Similarly, to assess the significance of enrichment for annotation it uses LRT with $\mathrm{H}_{0}$ : $\mathrm{q}_{00}=\mathrm{q}_{11}$, versus $\mathrm{H} 1$ : $\mathrm{q}_{00} \neq \mathrm{q}_{11}$. GPA also calculates the standard errors for the model parameters along with their covariance matrix based on an empirically observed information matrix.

After estimating the parameters, GPA assigns each SNP four posterior probabilities (PP) (estimated values of $\left.\left\{\pi_{00}, \pi_{10}, \pi_{01}, \pi_{11}\right\}\right)$ and controls for false discovery based on the local false discovery rate (FDR). The local FDR is defined as the probability that the jth SNP is either not associated with any trait $\left(\mathrm{Fdr}_{0}\right)$ or is associated with the first trait $\left(\mathrm{Fdr}_{1}\right)$, second trait $\left(\mathrm{Fdr}_{2}\right)$ or both trait $\left(\mathrm{Fdr}_{1,2}\right)$ given its $P$ value and annotation information.

$$
\begin{aligned}
& F_{d r}\left(p_{j 1}, p_{j 2}, A\right)=\operatorname{Pr}\left(Z j_{00}=1 \mid p_{j 1}, p_{j 2}, A\right) \\
& F_{r_{1}}\left(p_{j 1}, p_{j 2}, A\right)=\operatorname{Pr}\left(Z j_{00}+Z j_{01}=1 \mid p_{j 1}, p_{j 2}, A\right) \\
& F_{r_{2}}\left(p_{j 1}, p_{j 2}, A\right)=\operatorname{Pr}\left(Z j_{00}+Z j_{10}=1 \mid p_{j 1}, p_{j 2}, A\right) \\
& F_{r_{1,2}}\left(p_{j 1}, p_{j 2}, A\right)=\operatorname{Pr}\left(Z j_{00}+Z j_{01}+Z j_{10}=1 \mid p_{j 1}, p_{j 2}, A\right)
\end{aligned}
$$

Finally, to infer associations at the variant level and to control the FDR at 5\% we then select those SNPs with any of the four PP $>95 \%$ and FDR $<0.05$. From the selected set, SNPs that achieve PP $>95 \%$ under the PP categories of $\left\{\pi_{00}, \pi_{10}, \pi_{01}, \pi_{11}\right\}$ are concluded to be associated with neither, first, second or both traits. In our study, we conducted all the tests after controlling for FDR at 0.05 level and used 10,000 EM iterations.

Pathway analysis. Pathway analysis elucidates the underlying biological processes in which genes are related by common functionality. To detect such relationships of the identified pleiotropic genetic loci in our study, we used QIAGEN's IPA tool (https://digitalinsights.qiagen.com/). IPA is a web-based software application that allows analysis of data obtained from several sequencing platforms. IPA enables for targeted search of information on genes, proteins, chemicals, diseases, and drugs. IPA's data analysis provides clarity in understanding the significance of data or targets of interest in relation to larger biological systems. Statistical significance of overrepresented canonical pathways was determined using Fisher's exact test after adjustment for multiple testing using the Benjamini-Hochberg method. Statistical significance was based on $P<0.05$ in pathways with at least two molecules.

Colocalization analysis. To evaluated whether birthweight and childhood obesity traits share common genetic causal variant(s) in a region, we performed colocalization analysis for each of the 19 pleiotropic loci using the European ancestry GWAS summary statistics. For each genetic locus, the lead SNP with the highest posterior probability of joint association with birthweight and childhood obesity traits as well as SNPs within $500 \mathrm{~kb}$ window on either side of the lead SNP defined a colocalization region. Analysis was performed using the coloc R package $e^{47}$.

Ethical approval. The NIH Office of Human Subjects Research Program granted the study an exemption from IRB review (OHSRP ID Number: 18-NICHD-00412) per 45 CFR 46 and NIH policy for the use of specimens/data.

\section{Data availability}

The data analyzed in this study are available online. Table S1 lists the URL of the data sources. 
Received: 10 June 2020; Accepted: 9 December 2020

Published online: 08 January 2021

\section{References}

1. de Onis, M. \& Blossner, M. Prevalence and trends of overweight among preschool children in developing countries. Am. J. Clin. Nutr. 72, 1032-1039. https://doi.org/10.1093/ajcn/72.4.1032 (2000).

2. Kumar, S. \& Kelly, A. S. Review of childhood obesity: from epidemiology, etiology, and comorbidities to clinical assessment and treatment. Mayo Clin. Proc. 92, 251-265. https://doi.org/10.1016/j.mayocp.2016.09.017 (2017).

3. Ogden, C. L. et al. Prevalence of overweight and obesity in the United States, 1999-2004. JAMA 295, 1549-1555. https://doi.org/ 10.1001/jama.295.13.1549 (2006).

4. Elks, C. E. et al. Variability in the heritability of body mass index: a systematic review and meta-regression. Front. Endocrinol. (Lausanne) 3, 29. https://doi.org/10.3389/fendo.2012.00029 (2012).

5. Herrera, B. M., Keildson, S. \& Lindgren, C. M. Genetics and epigenetics of obesity. Maturitas 69, 41-49. https://doi.org/10.1016/j. maturitas.2011.02.018 (2011).

6. Wardle, J., Carnell, S., Haworth, C. M. \& Plomin, R. Evidence for a strong genetic influence on childhood adiposity despite the force of the obesogenic environment. Am. J. Clin. Nutr. 87, 398-404. https://doi.org/10.1093/ajcn/87.2.398 (2008).

7. Silventoinen, K., Rokholm, B., Kaprio, J. \& Sorensen, T. I. The genetic and environmental influences on childhood obesity: a systematic review of twin and adoption studies. Int. J. Obes. (Lond.) 34, 29-40. https://doi.org/10.1038/ijo.2009.177 (2010).

8. Tounian, P. Programming towards childhood obesity. Ann. Nutr. Metab. 58(Suppl 2), 30-41. https://doi.org/10.1159/000328038 (2011).

9. Dietz, W. H. Critical periods in childhood for the development of obesity. Am. J. Clin. Nutr. 59, 955-959. https://doi.org/10.1093/ ajcn/59.5.955 (1994).

10. Hui, L. L. et al. Birth weight, infant growth, and childhood body mass index: Hong Kong's children of 1997 birth cohort. Arch. Pediatr. Adolesc. Med. 162, 212-218. https://doi.org/10.1001/archpediatrics.2007.62 (2008).

11. Salsberry, P. J. \& Reagan, P. B. Dynamics of early childhood overweight. Pediatrics 116, 1329-1338. https://doi.org/10.1542/peds. 2004-2583 (2005).

12. Whitaker, R. C., Wright, J. A., Pepe, M. S., Seidel, K. D. \& Dietz, W. H. Predicting obesity in young adulthood from childhood and parental obesity. N. Engl. J. Med. 337, 869-873. https://doi.org/10.1056/NEJM199709253371301 (1997).

13. Abe, Y. et al. Lower birth weight associated with current overweight status is related with the metabolic syndrome in obese Japanese children. Hypertens. Res. 30, 627-634. https://doi.org/10.1291/hypres.30.627 (2007).

14. Baird, J. et al. Being big or growing fast: systematic review of size and growth in infancy and later obesity. BMJ 331, 929. https:// doi.org/10.1136/bmj.38586.411273.E0 (2005).

15. Binkin, N. J., Yip, R., Fleshood, L. \& Trowbridge, F. L. Birth weight and childhood growth. Pediatrics 82, 828-834 (1988).

16. Hirschler, V., Bugna, J., Roque, M., Gilligan, T. \& Gonzalez, C. Does low birth weight predict obesity/overweight and metabolic syndrome in elementary school children?. Arch. Med. Res 39, 796-802. https://doi.org/10.1016/j.arcmed.2008.08.003 (2008).

17. Mei, Z., Grummer-Strawn, L. M. \& Scanlon, K. S. Does overweight in infancy persist through the preschool years? An analysis of CDC pediatric nutrition surveillance system data. Soz. Praventivmed. 48, 161-167. https://doi.org/10.1007/s00038-003-2022-x (2003).

18. Qiao, Y. et al. Birth weight and childhood obesity: a 12-country study. Int. J. Obes. Suppl. 5, S74-79. https://doi.org/10.1038/ijosup. 2015.23 (2015).

19. Sacco, M. R., de Castro, N. P., Euclydes, V. L., Souza, J. M. \& Rondo, P. H. Birth weight, rapid weight gain in infancy and markers of overweight and obesity in childhood. Eur. J. Clin. Nutr. 67, 1147-1153. https://doi.org/10.1038/ejcn.2013.183 (2013).

20. Stettler, N., Zemel, B. S., Kumanyika, S. \& Stallings, V. A. Infant weight gain and childhood overweight status in a multicenter, cohort study. Pediatrics 109, 194-199. https://doi.org/10.1542/peds.109.2.194 (2002).

21. Peng, S. et al. Genetic regulation of the placental transcriptome underlies birth weight and risk of childhood obesity. PLoS Genet. 14, e1007799. https://doi.org/10.1371/journal.pgen.1007799 (2018).

22. Bulik-Sullivan, B. et al. An atlas of genetic correlations across human diseases and traits. Nat. Genet. 47, 1236-1241. https://doi. org/10.1038/ng.3406 (2015).

23. Cross-Disorder Group of the Psychiatric Genomics C. Identification of risk loci with shared effects on five major psychiatric disorders: a genome-wide analysis. Lancet 381, 1371-1379. https://doi.org/10.1016/S0140-6736(12)62129-1 (2013).

24. Horikoshi, M. et al. New loci associated with birth weight identify genetic links between intrauterine growth and adult height and metabolism. Nat. Genet. 45, 76-82. https://doi.org/10.1038/ng.2477 (2013).

25. Sakoda, L. C., Jorgenson, E. \& Witte, J. S. Turning of COGS moves forward findings for hormonally mediated cancers. Nat. Genet. 45, 345-348. https://doi.org/10.1038/ng.2587 (2013).

26. Vattikuti, S., Guo, J. \& Chow, C. C. Heritability and genetic correlations explained by common SNPs for metabolic syndrome traits. PLoS Genet. 8, e1002637. https://doi.org/10.1371/journal.pgen.1002637 (2012).

27. MacArthur, J. et al. The new NHGRI-EBI Catalog of published genome-wide association studies (GWAS Catalog). Nucleic Acids Res. 45, D896-D901. https://doi.org/10.1093/nar/gkw1133 (2017).

28. Sivakumaran, S. et al. Abundant pleiotropy in human complex diseases and traits. Am. J. Hum. Genet. 89, 607-618. https://doi. org/10.1016/j.ajhg.2011.10.004 (2011).

29. Nicolae, D. L. et al. Trait-associated SNPs are more likely to be eQTLs: annotation to enhance discovery from GWAS. PLoS Genet. 6, e1000888. https://doi.org/10.1371/journal.pgen.1000888 (2010).

30. Schork, A. J. et al. All SNPs are not created equal: genome-wide association studies reveal a consistent pattern of enrichment among functionally annotated SNPs. PLoS Genet. 9, e1003449. https://doi.org/10.1371/journal.pgen.1003449 (2013).

31. Yang, J. et al. Genome partitioning of genetic variation for complex traits using common SNPs. Nat. Genet. 43, 519-525. https:// doi.org/10.1038/ng.823 (2011)

32. Andreassen, O. A. et al. Improved detection of common variants associated with schizophrenia by leveraging pleiotropy with cardiovascular-disease risk factors. Am. J. Hum. Genet. 92, 197-209. https://doi.org/10.1016/j.ajhg.2013.01.001 (2013).

33. Bouchard, C. Childhood obesity: are genetic differences involved?. Am. J. Clin. Nutr. 89, 1494S-1501S. https://doi.org/10.3945/ ajcn.2009.27113C (2009).

34. Chesi, A. \& Grant, S. F. A. The genetics of pediatric obesity. Trends Endocrinol. Metab. 26, 711-721. https://doi.org/10.1016/j.tem. 2015.08.008 (2015).

35. Clausson, B., Lichtenstein, P. \& Cnattingius, S. Genetic influence on birthweight and gestational length determined by studies in offspring of twins. BJOG 107, 375-381. https://doi.org/10.1111/j.1471-0528.2000.tb13234.x (2000).

36. Warrington, N. M. et al. Maternal and fetal genetic effects on birth weight and their relevance to cardio-metabolic risk factors. Nat. Genet. 51, 804-814. https://doi.org/10.1038/s41588-019-0403-1 (2019).

37. Bradfield, J. P. et al. A genome-wide association meta-analysis identifies new childhood obesity loci. Nat. Genet. Nat. Genet. 44, 526-531. https://doi.org/10.1038/ng.2247 (2012).

38. Couto Alves, A. et al. GWAS on longitudinal growth traits reveals different genetic factors influencing infant, child, and adult BMI. Sci. Adv. 5, 3095. https://doi.org/10.1126/sciadv.aaw3095 (2019). 
39. Felix, J. F. et al. Genome-wide association analysis identifies three new susceptibility loci for childhood body mass index. Hum. Mol. Genet. 25, 389-403. https://doi.org/10.1093/hmg/ddv472 (2016).

40. Helgeland, O. et al. Genome-wide association study reveals dynamic role of genetic variation in infant and early childhood growth. Nat. Commun. 10, 4448. https://doi.org/10.1038/s41467-019-12308-0 (2019).

41. Chung, D., Yang, C., Li, C., Gelernter, J. \& Zhao, H. GPA: a statistical approach to prioritizing GWAS results by integrating pleiotropy and annotation. PLoS Genet. 10, e1004787. https://doi.org/10.1371/journal.pgen.1004787 (2014).

42. Kircher, M. et al. A general framework for estimating the relative pathogenicity of human genetic variants. Nat. Genet. 46, 310-315. https://doi.org/10.1038/ng.2892 (2014).

43. Consortium, G. T. The genotype-tissue expression (GTEx) project. Nat. Genet. 45, 580-585. https://doi.org/10.1038/ng.2653 (2013).

44. Kumar, S., Ambrosini, G. \& Bucher, P. SNP2TFBS-a database of regulatory SNPs affecting predicted transcription factor binding site affinity. Nucleic Acids. Res. 45, D139-D144. https://doi.org/10.1093/nar/gkw1064 (2017).

45. Pickrell, J. K. Joint analysis of functional genomic data and genome-wide association studies of 18 human traits. Am. J. Hum. Genet. 94, 559-573. https://doi.org/10.1016/j.aihg.2014.03.004 (2014).

46. Bradfield, J. P. et al. A trans-ancestral meta-analysis of genome-wide association studies reveals loci associated with childhood obesity. Hum. Mol. Genet. 28, 3327-3338. https://doi.org/10.1093/hmg/ddz161 (2019).

47. Giambartolomei, C. et al. Bayesian test for colocalisation between pairs of genetic association studies using summary statistics. PLoS Genet. 10, e1004383. https://doi.org/10.1371/journal.pgen.1004383 (2014).

48. Pickrell, J. K. et al. Detection and interpretation of shared genetic influences on 42 human traits. Nat. Genet. 48, 709-717. https:// doi.org/10.1038/ng.3570 (2016).

49. Stearns, F. W. One hundred years of pleiotropy: a retrospective. Genetics 186, 767-773. https://doi.org/10.1534/genetics.110.122549 (2010).

50. Tekola-Ayele, F., Lee, A., Workalemahu, T. \& Sanchez-Pozos, K. Shared genetic underpinnings of childhood obesity and adult cardiometabolic diseases. Hum. Genom. 13, 17. https://doi.org/10.1186/s40246-019-0202-x (2019).

51. Tekola-Ayele, F. et al. Genetic overlap between birthweight and adult cardiometabolic diseases has implications for genomic medicine. Sci. Rep. 9, 4076. https://doi.org/10.1038/s41598-019-40834-w (2019).

52. He, X. \& Zhang, J. Toward a molecular understanding of pleiotropy. Genetics 173, 1885-1891. https://doi.org/10.1534/genetics. 106.060269 (2006).

53. Beaumont, R. N. et al. Genome-wide association study of offspring birth weight in 86577 women identifies five novel loci and highlights maternal genetic effects that are independent of fetal genetics. Hum. Mol. Genet. 27, 742-756. https://doi.org/10.1093/ $\mathrm{hmg} / \mathrm{ddx} 429$ (2018).

54. Horikoshi, M. et al. Genome-wide associations for birth weight and correlations with adult disease. Nature 538, 248-252. https:// doi.org/10.1038/nature19806 (2016).

55. Zhang, G. et al. Genetic associations with gestational duration and spontaneous preterm birth. N. Engl. J. Med. 377, 1156-1167. https://doi.org/10.1056/NEJMoa1612665 (2017).

56. Chu, A. Y. et al. Multiethnic genome-wide meta-analysis of ectopic fat depots identifies loci associated with adipocyte development and differentiation. Nat. Genet. 49, 125-130. https://doi.org/10.1038/ng.3738 (2017).

57. Gao, H. et al. Early B cell factor 1 regulates adipocyte morphology and lipolysis in white adipose tissue. Cell Metab. 19, 981-992. https://doi.org/10.1016/j.cmet.2014.03.032 (2014).

58. Jimenez, M. A., Akerblad, P., Sigvardsson, M. \& Rosen, E. D. Critical role for Ebf1 and Ebf2 in the adipogenic transcriptional cascade. Mol. Cell. Biol. 27, 743-757. https://doi.org/10.1128/MCB.01557-06 (2007).

59. Longo, M. et al. Adipose tissue dysfunction as determinant of obesity-associated metabolic complications. Int. J. Mol. Sci. 20, 1. https://doi.org/10.3390/ijms20092358 (2019).

60. International Consortium for Blood Pressure Genome-Wide Association, S. et al. Genetic variants in novel pathways influence blood pressure and cardiovascular disease risk. Nature 478, 103-109. https://doi.org/10.1038/nature10405 (2011).

61. Singh, A. et al. Gene by stress genome-wide interaction analysis and path analysis identify EBF1 as a cardiovascular and metabolic risk gene. Eur. J. Hum. Genet. 23, 854-862. https://doi.org/10.1038/ejhg.2014.189 (2015).

62. Vogelezang, S. et al. Novel loci for childhood body mass index and shared heritability with adult cardiometabolic traits. PLoS Genet. 16, e1008718. https://doi.org/10.1371/journal.pgen.1008718 (2020).

63. Griffin, M. J. et al. Early B-cell factor-1 (EBF1) is a key regulator of metabolic and inflammatory signaling pathways in mature adipocytes. J. Biol. Chem. 288, 35925-35939. https://doi.org/10.1074/jbc.M113.491936 (2013).

64. Choi, J. H. et al. LMBR1L regulates lymphopoiesis through Wnt/beta-catenin signaling. Science 364, 1. https://doi.org/10.1126/ science.aau0812 (2019).

65. Barker, N. The canonical Wnt/beta-catenin signalling pathway. Methods Mol. Biol. 468, 5-15. https://doi.org/10.1007/978-1-59745249-6_1 (2008).

66. Chan, E. F., Gat, U., McNiff, J. M. \& Fuchs, E. A common human skin tumour is caused by activating mutations in beta-catenin. Nat. Genet. 21, 410-413. https://doi.org/10.1038/7747 (1999).

67. Deng, C., Dai, R., Li, X. \& Liu, F. Genetic variation frequencies in Wilms' tumor: a meta-analysis and systematic review. Cancer Sci. 107, 690-699. https://doi.org/10.1111/cas.12910 (2016).

68. Xia, J. et al. beta-Catenin mutation and its nuclear localization are confirmed to be frequent causes of Wnt signaling pathway activation in pilomatricomas. J. Dermatol. Sci. 41, 67-75. https://doi.org/10.1016/j.jdermsci.2005.09.005 (2006).

69. Teo, J. L. \& Kahn, M. The Wnt signaling pathway in cellular proliferation and differentiation: a tale of two coactivators. Adv. Drug Deliv. Rev. 62, 1149-1155. https://doi.org/10.1016/j.addr.2010.09.012 (2010).

70. Christodoulides, C., Lagathu, C., Sethi, J. K. \& Vidal-Puig, A. Adipogenesis and WNT signalling. Trends Endocrinol. Metab. 20, 16-24. https://doi.org/10.1016/j.tem.2008.09.002 (2009).

71. Ross, S. E. et al. Inhibition of adipogenesis by Wnt signaling. Science 289, 950-953. https://doi.org/10.1126/science.289.5481.950 (2000).

72. Cartwright, J. E., Fraser, R., Leslie, K., Wallace, A. E. \& James, J. L. Remodelling at the maternal-fetal interface: relevance to human pregnancy disorders. Reproduction 140, 803-813. https://doi.org/10.1530/REP-10-0294 (2010).

73. Nayeem, S. B., Arfuso, F., Dharmarajan, A. \& Keelan, J. A. Role of Wnt signalling in early pregnancy. Reprod. Fertil. Dev. 28, 525-544. https://doi.org/10.1071/RD14079 (2016).

74. Red-Horse, K. et al. Trophoblast differentiation during embryo implantation and formation of the maternal-fetal interface. J. Clin. Investig. 114, 744-754. https://doi.org/10.1172/JCI22991 (2004).

75. Comuzzie, A. G. et al. Novel genetic loci identified for the pathophysiology of childhood obesity in the Hispanic population. PLoS ONE 7, e51954. https://doi.org/10.1371/journal.pone.0051954 (2012).

76. Morgan, A. R. et al. Obesity and diabetes genes are associated with being born small for gestational age: results from the Auckland birthweight collaborative study. BMC Med. Genet. 11, 125. https://doi.org/10.1186/1471-2350-11-125 (2010).

77. Chen, X., Liu, Z. \& Xu, J. The cooperative function of nuclear receptor coactivator 1 (NCOA1) and NCOA3 in placental development and embryo survival. Mol. Endocrinol. 24, 1917-1934. https://doi.org/10.1210/me.2010-0201 (2010).

78. Kim, S. C., Park, M. N., Lee, Y. J., Joo, J. K. \& An, B. S. Interaction of steroid receptor coactivators and estrogen receptors in the human placenta. J. Mol. Endocrinol. 56, 239-247. https://doi.org/10.1530/JME-15-0248 (2016). 
79. Rollins, D. A., Coppo, M. \& Rogatsky, I. Minireview: nuclear receptor coregulators of the p160 family: insights into inflammation and metabolism. Mol. Endocrinol. 29, 502-517. https://doi.org/10.1210/me.2015-1005 (2015).

80. Ruan, X. Z., Varghese, Z., Powis, S. H. \& Moorhead, J. F. Nuclear receptors and their coregulators in kidney. Kidney Int. 68, 2444-2461. https://doi.org/10.1111/j.1523-1755.2005.00721.x (2005).

81. Kovats, S. Estrogen receptors regulate innate immune cells and signaling pathways. Cell. Immunol. 294, 63-69. https://doi.org/10. 1016/j.cellimm.2015.01.018 (2015).

82. Marti, A., Marcos, A. \& Martinez, J. A. Obesity and immune function relationships. Obes. Rev. 2, 131-140. https://doi.org/10. 1046/j.1467-789x.2001.00025.x (2001).

83. Tam, C. S. et al. An early inflammatory gene profile in visceral adipose tissue in children. Int. J. Pediatr. Obes. 6, e360-363. https:// doi.org/10.3109/17477166.2011.575152 (2011).

84. Pairault, J., Quignard-Boulange, A., Dugail, I. \& Lasnier, F. Differential effects of retinoic acid upon early and late events in adipose conversion of 3T3 preadipocytes. Exp. Cell Res. 177, 27-36. https://doi.org/10.1016/0014-4827(88)90022-5 (1988).

85. Sato, M., Hiragun, A. \& Mitsui, H. Preadipocytes possess cellular retinoid binding proteins and their differentiation is inhibited by retinoids. Biochem. Biophys. Res. Commun. 95, 1839-1845. https://doi.org/10.1016/s0006-291x(80)80113-6 (1980).

86. Schwarz, E. J., Reginato, M. J., Shao, D., Krakow, S. L. \& Lazar, M. A. Retinoic acid blocks adipogenesis by inhibiting C/EBPbetamediated transcription. Mol. Cell. Biol. 17, 1552-1561. https://doi.org/10.1128/mcb.17.3.1552 (1997).

87. Kowianski, P. et al. BDNF: a key factor with multipotent impact on brain signaling and synaptic plasticity. Cell. Mol. Neurobiol. 38, 579-593. https://doi.org/10.1007/s10571-017-0510-4 (2018).

88. Cordeira, J. \& Rios, M. Weighing in the role of BDNF in the central control of eating behavior. Mol. Neurobiol. 44, 441-448. https:// doi.org/10.1007/s12035-011-8212-2 (2011).

89. Xu, B. et al. Brain-derived neurotrophic factor regulates energy balance downstream of melanocortin-4 receptor. Nat. Neurosci. 6, 736-742. https://doi.org/10.1038/nn1073 (2003).

90. Rothman, S. M., Griffioen, K. J., Wan, R. \& Mattson, M. P. Brain-derived neurotrophic factor as a regulator of systemic and brain energy metabolism and cardiovascular health. Ann. N. Y. Acad. Sci. 1264, 49-63. https://doi.org/10.1111/j.1749-6632.2012.06525.x (2012).

91. Han, J. C., Muehlbauer, M. J., Cui, H. N., Newgard, C. B. \& Haqq, A. M. Lower brain-derived neurotrophic factor in patients with prader-willi syndrome compared to obese and lean control subjects. J. Clin. Endocrinol. Metab. 95, 3532-3536. https://doi.org/10. 1210/jc.2010-0127 (2010).

92. Yeo, G. S. et al. A de novo mutation affecting human TrkB associated with severe obesity and developmental delay. Nat. Neurosci. 7, 1187-1189. https://doi.org/10.1038/nn1336 (2004).

93. Chen, W., McDonnell, S. K., Thibodeau, S. N., Tillmans, L. S. \& Schaid, D. J. Incorporating functional annotations for fine-mapping causal variants in a bayesian framework using summary statistics. Genetics 204, 933-958. https://doi.org/10.1534/genetics.116. $188953(2016)$.

94. Maurano, M. T. et al. Systematic localization of common disease-associated variation in regulatory DNA. Science 337, 1190-1195. https://doi.org/10.1126/science.1222794 (2012).

95. Thurman, R. E. et al. The accessible chromatin landscape of the human genome. Nature 489, 75-82. https://doi.org/10.1038/natur e11232 (2012).

\section{Author contributions}

F.T.-A. and S.C. conceived and designed this study; S.C. gathered and managed data; S.C. analyzed the data; S.C. wrote the draft paper; F.T.-A. supervised the work and interpreted the results. M.O. provided critical intellectual content. All authors approved the final manuscript.

\section{Funding}

This research was supported by the Intramural Research Program of the Eunice Kennedy Shriver National Institute of Child Health and Human Development, National Institutes of Health. Additional support was obtained from the National Institute on Minority Health and Health Disparities and the National Institute of Diabetes and Digestive and Kidney Diseases. This work utilized the computational resources of the NIH HPC Biowulf cluster (http://hpc.nih.gov).

\section{Competing interests}

The authors declare no competing interests.

\section{Additional information}

Supplementary Information The online version contains supplementary material available at https:/doi.org/ 10.1038/s41598-020-80084-9.

Correspondence and requests for materials should be addressed to F.T.-A.

Reprints and permissions information is available at www.nature.com/reprints.

Publisher's note Springer Nature remains neutral with regard to jurisdictional claims in published maps and institutional affiliations.

(c) (i) Open Access This article is licensed under a Creative Commons Attribution 4.0 International License, which permits use, sharing, adaptation, distribution and reproduction in any medium or format, as long as you give appropriate credit to the original author(s) and the source, provide a link to the Creative Commons licence, and indicate if changes were made. The images or other third party material in this article are included in the article's Creative Commons licence, unless indicated otherwise in a credit line to the material. If material is not included in the article's Creative Commons licence and your intended use is not permitted by statutory regulation or exceeds the permitted use, you will need to obtain permission directly from the copyright holder. To view a copy of this licence, visit http://creativecommons.org/licenses/by/4.0/.

This is a U.S. Government work and not under copyright protection in the US; foreign copyright protection may apply 2021 